\section{Improved hybrid MMSE detection for turbo-trellis-coded modulation-assisted multi-user OFDM systems}

\author{
M. Jiang and L. Hanzo
}

A genetic algorithm assisted minimum mean-square error (MMSE) multi-user detector (MUD) is proposed for a turbo-trellis-coded modulation-based space division multiple access aided orthogonal frequency division multiplexing system. The simulation results show that the system employing the concatenated MMSE-GA MUD was capable of achieving a similar performance to that attained by its optimum counterpart employing the maximum likelihood MUD, while maintaining a significantly lower complexity, especially when supporting a high number of users.

Introduction: Genetic algorithm (GA) [1] aided transceiver research has been documented in the context of code division multiple access (CDMA) systems in [2, 3]. We present the design of a different transceiver, namely minimum mean-square error (MMSE) multi-user detector (MUD) assisted space division multiple access (SDMA) [4] orthogonal frequency division multiplexing (OFDM) system invoking turbo-trellis-coded modulation (TTCM) [5]. We demonstrate that the proposed concatenated MMSE-GA assisted TTCM-coded SDMAOFDM system is capable of achieving a similar performance to that attained by its optimum maximum likelihood (ML) MUD assisted counterpart at a significantly lower computational complexity, especially at high user loads. The schematic of the proposed uplink system is shown in Fig. 1. The SDMA system's philosophy is that $L>1$ users are supported within the same time slot and frequency slot by differentiating them with the aid of their unique, user-specific channel impulse responses (CIRs). The information bits of the mobile users are encoded into symbols by the TTCM encoders and transmitted over the SDMA uplink channel after OFDM modulation. At the base station (BS), where $P$ number of receiver antenna elements are employed, the OFDM demodulated received signals $x_{p}(p=1 \ldots P)$ are forwarded to the proposed concatenated MMSE-GA MUD for separating the different users' signals and then decoded by the TTCM decoders. The rationale of using TTCM channel coding is that it achieves the highest coding gain in the family of the coded modulation schemes [5] without any bandwidth expansion, since it accommodates the parity bits by increasing the number of bits per symbol used by the modem.

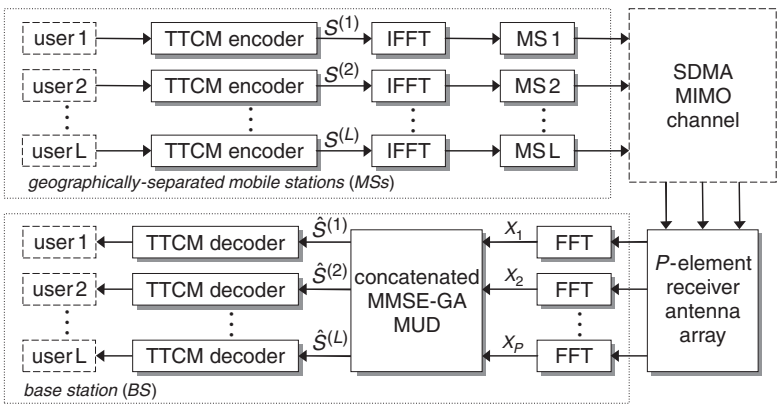

Fig. 1 Schematic of MMSE-GA-concatenated multi-user detected SDMAOFDM uplink system

Optimisation metric for GA MUD: In our GA MUD, an optimum ML-based decision metric is proposed for detecting the estimated transmitted symbol vector $\hat{s}_{\mathrm{GA}}$, while dispensing with a full-complexity ML search. The branch decision metric for the $p$ th receiver antenna, namely the antenna-specific objective function, is defined by:

$$
\Omega_{p}(\boldsymbol{s})=\left|x_{p}-\boldsymbol{H}_{p} \boldsymbol{s}\right|^{2}
$$

where $x_{p}$ is the OFDM-demodulated symbol received at the $p$ th receiver at a specific OFDM subcarrier, and $\boldsymbol{H}_{p}$ is the $p$ th row of the $P \times L$ frequency-domain channel transfer factor matrix $\boldsymbol{H}$. Our task is to find the specific estimated transmitted $L$-symbol vector $\hat{\boldsymbol{s}}_{\mathrm{GA}_{p}}$ of the $L$ users, which minimises the metric given in (1). However, we have $P$ different metrics for the $P$ number of receiver antennas, since the CIRs of each antenna are statistically independent and hence the $L$-symbol vector considered optimum at a specific antenna may not be considered optimum at the others, yielding the following decision conflict: $\arg \left\{\min _{s}\left[\Omega_{i}(\boldsymbol{s})\right]\right\}=\hat{\boldsymbol{s}}_{\mathrm{GA}_{i}} \neq \hat{\boldsymbol{s}}_{\mathrm{GA}_{i}}=\arg \left\{\min _{s}\left[\Omega_{j}(\boldsymbol{s})\right]\right\}, \quad$ where $\quad \forall i, \quad j \in$ $\{1, \ldots, P\}, i \neq j$. For the sake of solving this so-called multi-objective optimisation problem, we may convert the $P$ number of branch metrics into a 'joint' metric as follows [6]:

$$
\Omega(s)=\sum_{p=1}^{P} \Omega_{p}(s)
$$

Therefore, the ultimate decision rule is to find the specific $\hat{\boldsymbol{s}}_{\mathrm{GA}}$ that minimises $\Omega(s)$ in (2).

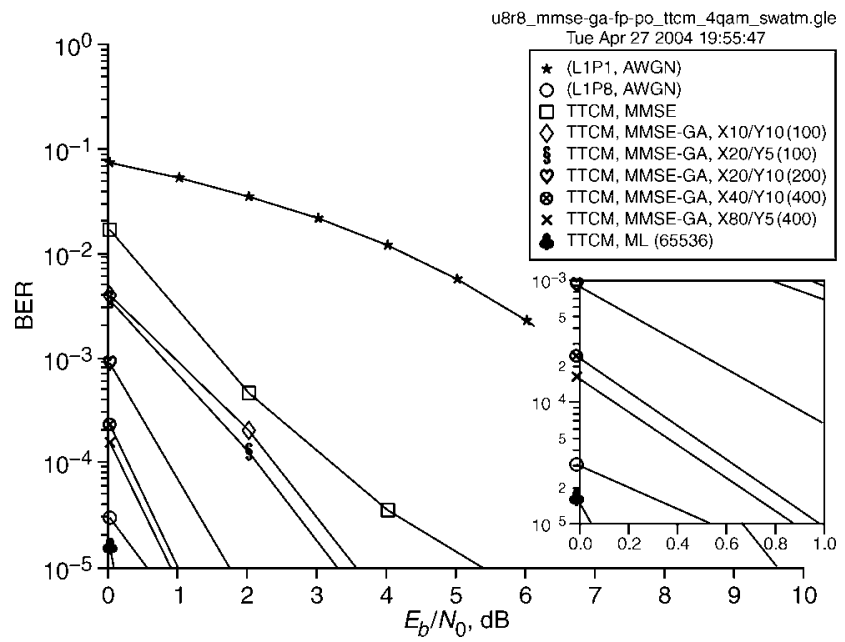

Fig. 2 BER against $E_{b} / N_{0}$ performance of TTCM-assisted MMSE-GA-SDMA-OFDM system employing a $4 Q A M$ scheme for transmission over SWATM channel, where eight users $(L=8)$ are supported with aid of eight receiver antenna elements $(P=8)$

Concatenated MMSE-GA multi-user detection: To avoid an inefficient, entirely random search, it is beneficial to supply the GA MUD with a good initial guess of the estimated transmitted $L$-symbol vectors to be detected. A low-cost design option may include the simple MMSE-based OFDM MUD's $L$-user output vector in the GA's initial population, which imposes a fairly low complexity compared to the interference-cancellation based schemes of [4]. In the MMSE MUD the estimated $L$-user signal vector $\hat{\boldsymbol{s}}_{\mathrm{MMSE}}$ is obtained as follows [4]: $\hat{\boldsymbol{s}}_{\mathrm{MMSE}}=\left(\boldsymbol{H} \boldsymbol{H}^{H}+\sigma_{n}^{2} \boldsymbol{I}\right)^{-1} \boldsymbol{H} \boldsymbol{x}$, where $\boldsymbol{x}$ is the length $P$ received signal vector, $\boldsymbol{I}$ is the identity matrix and $\sigma_{n}^{2}$ is the AWGN noise variance. Therefore, the $L$-symbol output of the MMSE MUD, namely $\hat{\boldsymbol{s}}_{\text {MMSE, }}$, may be included in the GA MUD's so-called initial population, which contains $X$ number of $L$-symbol individuals, resulting in the initial generation. Then the GA selects some of the $L$-symbol candidates from the $X$ individuals for creating a mating pool of $T$ number of parent vectors. The individual-selection strategy used is based on the concept of the so-called Pareto optimality [1]. This strategy retains the so-called non-dominated individuals and ignores the so-called dominated individuals. More specifically, the $u$ th individual is considered to be dominated by the $v$ th individual, if we have: $\forall_{i} \in\{1, \ldots, L\}: \Omega_{i}\left(\tilde{\boldsymbol{s}}_{v}^{(y)}\right) \leq \Omega_{i}\left(\tilde{\boldsymbol{s}}_{u}^{(y)}\right) \quad \Lambda \exists j \in\{1, \ldots, L\}: \Omega_{j}\left(\tilde{\mathbf{s}}_{v}^{(y)}\right)>$ $\Omega_{j}\left(\tilde{s}_{u}^{(y)}\right)$, where $y$ denotes the generation index. All the non-dominated individuals are then selected and placed in the mating pool having a size of $2<T \leq X$. Two $L$-symbol individuals in the mating pool are then selected as parents according to the so-called fitnessproportionate selection scheme based on their corresponding fitness values, which are calculated with the aid of the windowing-mapping technique as: $f_{i}^{(y)}=\Omega_{T}^{(y)}-\Omega\left(\tilde{\boldsymbol{s}}_{i}^{(y)}\right)+c$, where $\Omega_{T}^{(y)}=\max _{t \in\{1, \ldots, T\}}$ $\left\{\Omega\left(\tilde{\boldsymbol{s}}_{t}^{(y)}\right)\right\}$ is the maximum objective score (the output of (2) is called the objective score of the specific individual evaluated) achieved by evaluating all the $T$ number of individuals in the mating pool at the $y$ th generation, and $c$ is a small positive constant used for ensuring the positiveness of $f_{i}^{(y)}$. Then the fitness-proportionate selection probability $p_{i}$ of the $i$ th individual can be formulated as: $p_{i}=f_{i}^{(y)} /$ $\sum_{j=1}^{T} f_{j}^{(y)}$. The selection process is then repeated, which is followed by the crossover, mutation and elitism operations [3], until a new population is created. Finally, the GA terminates after $(Y-1)$ number of generations and thus the $L$-symbol individual having the highest 
fitness value will be output as the detected $L$-user transmitted symbol vector at the specific OFDM subcarrier considered.

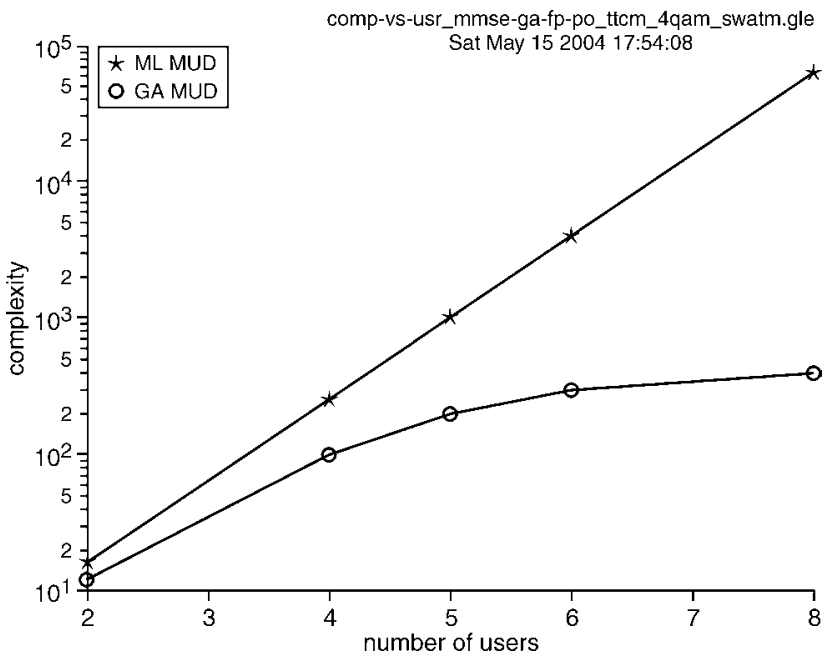

Fig. 3 Comparison of MUD complexity, in terms of number of metric evaluations, against number of users performance of $4 Q A M T T C M-M M$ SE-GA-SDMA-OFDM and TTCM-ML-SDMA-OFDM systems

Number of receiver antenna elements employed is equivalent to number of users supported, i.e. $L=P$

Simulation results: In our simulations, the short wireless asynchronous transfer mode (SWATM) channel model of p. 78 of [4] was employed, while the normalised Doppler frequency was $f_{d}^{\prime}=1.235 \times 10^{-5}$. The fading envelope was assumed to be 'OFDM symbol-invariant' [4] and perfectly estimated. The bit error rate (BER) performance of the TTCM-MMSE-GA-SDMA-OFDM system is shown in Fig. 2. The BER performance of the MMSE- or ML-detected systems and that of the single-user scheme employing either a single receiver or invoking maximum ratio combining (MRC), when communicating over an AWGN channel are also provided for reference. It is observed from Fig. 2 that the GA-assisted system was capable of achieving a similar performance to that attained by the optimum TTCM-ML-SDMA-OFDM arrangement, provided that a sufficiently large population size $X$ and/or number of generations $Y$ were used. Furthermore, this was achieved at a significantly lower computational complexity than that of the ML-aided system, as demonstrated below. For the sake of simplicity, we only compare the GA MUD's complexity to that of the optimum ML MUD, since the simple MMSE MUD only provides a single initial solution and imposes a significantly lower complexity than that of its concatenated GA-aided counterpart. More specifically, we quantify the complexity in terms of the number of fitness metric evaluations associated with (1). For the ML MUD, $2^{\mathrm{mL}}$ number of metric computations have to be carried out for finding the optimum solution [4], where $m$ denotes the number of bits per symbol. By contrast, our proposed GA MUD requires a maximum of $X \times Y$ metric evaluations, as shown in round brackets in the legends of Fig. 2. Both the ML and GA-aided schemes are compared in terms of their complexity in Fig. 3. At a specific user load, we always select an appropriate GA-aided scheme for comparison, which suffers from less than $1 \mathrm{~dB} E_{b} / N_{0}$ loss at the BER of $10^{-5}$ compared to the ML-aided system. As shown in Fig. 3, the ML-aided system imposes an exponentially increasing complexity of the order of $\mathrm{O}\left(2^{m L}\right)$, when the number of users increases, while the complexity of the GA-aided system required for maintaining a near-optimum performance increases only slowly.

Conclusions: The proposed GA-assisted TTCM-aided MMSESDMA-OFDM system is capable of achieving a similar performance to that of the optimum ML-assisted TTCM-SDMA-OFDM system at a significantly lower computational complexity, achieving a complexity reduction in excess of a factor of 100 for $L=P=8$.

Acknowledgment: The financial support of the Mobile VCE, www.mobilevce.com, is gratefully acknowledged.

(C) IEE 2004

1 June 2004

Electronics Letters online no: 20045630

doi: 10.1049/el:20045630

M. Jiang and L. Hanzo (School of Electronics and Computer Science, University of Southampton, Southampton SO17 1BJ, United Kingdom)

\section{References}

1 Goldberg, D.E.: 'Genetic algorithms in search, optimization, and machine learning' (Addison-Wesley, Reading, MA, USA, 1989)

2 Abedi, S., and Tafazolli, R.: 'Genetically modified multiuser detection for code division multiple access systems', IEEE J. Sel. Areas Commun., 2002, 20, pp. 463-473

3 Hanzo, L., et al:: 'Single- and multi-carrier DS-CDMA: multi-user detection, space-time spreading, synchronisation and standards' (IEEE Press/John Wiley \& Sons Ltd., New York, USA, 2003)

4 Hanzo, L., et al.: 'OFDM and MC-CDMA for broadband multi-user communications, WLANs and broadcasting' (IEEE Press/John Wiley \& Sons Ltd., New York, USA, 2003)

5 Hanzo, L., Liew, T., and Yeap, B.: 'Turbo coding, turbo equalisation and space-time coding for transmission over fading channels' (IEEE Press/John Wiley \& Sons Ltd., New York, USA, 2002)

6 Fawer, U, and Aazhang, B. "A multiuser receiver for code division multiple access communications over multipath channels', IEEE Trans. Commun, 1995, 43, pp. 1556-1565 